\title{
Proximidades e convergências entre a Modelagem Matemática e o STEAM
}

\author{
Proximities and convergences between Mathematical Modeling and STEAM
}

\author{
José Ricardo Dolenga Coelho \\ Anderson Roges Teixeira Góes
}

\begin{abstract}
Resumo: Considerando a necessidade de práticas pedagógicas diferenciadas que possibilitem associar a realidade do estudante com outras áreas do conhecimento, para que conceitos/conteúdos científicos/escolares tenham maior significado, este estudo apresenta as proximidades e convergências entre as metodologias Modelagem Matemática e STEAM. Tal discussão mostra-se necessária, pois ao verificar pesquisas no Catálogo de Teses e Dissertações da Coordenação de Aperfeiçoamento de Pessoal de Nível Superior (CAPES), não foram identificados trabalhos envolvendo ambas metodologias. Com isso, este estudo apresenta elementos associando semelhanças nas etapas/fases indicadas por cada metodologia, o que possibilita ao estudante, mediado pelo professor, desenvolver modelos matemáticos e físicos para a resolução de situações-problema. Pelos estudos teóricos realizados, é possível considerar que as duas metodologias têm aproximações e podem ser inseridas nos processos de ensino e de aprendizagem de Matemática, possibilitando que o estudante seja o protagonista na construção de seu conhecimento, não somente de conceitos matemáticos, mas numa abordagem interdisciplinar.
\end{abstract}

Palavras-chave: Ensino e aprendizagem. Resolução de Problemas. Metodologias ativas. Tendências em Educação Matemática.

Abstract: Considering the need for differentiated pedagogical practices enabling the association of student's reality and other areas of knowledge, so that scientific/school concepts/content have greater meaning, this paper presents the approximation between methodologies Mathematical Modeling and STEAM. Such discussion is indispensable, because when researching the Catalog of Theses and Dissertations of the Coordination for the Improvement of Higher Education Personnel (CAPES), no studies involving both methodologies were identified. With this, research presents elements associating similarities in the stages/phases indicated by each methodology, which allow the student, mediated by the teacher, to develop mathematical and physical models to solve current problem situations. Based on the theoretical studies carried out, it is possible to consider that the two methodologies have similarities and can be inserted into the process of Mathemathics teaching and learning, allowing the student to be the protagonist in the construction of his

José Ricardo Dolenga Coelho Mestrando em Educação: Teoria e Prática de Ensino da Universidade Federal do Paraná (UFPR). Professor da Secretaria Municipal de Educação de Curitiba. Paraná, Brasil. iD orcid.org/0000-0002-6615-9319 $\triangle$ dolengacoelho@gmail.com

Anderson Roges Teixeira Góes Doutor em Métodos Numéricos em Engenharia. Professor do Programa de PósGraduação em Educação: Teoria e Prática de Ensino e do Programa de Pós-Graduação em Educação em Ciências e em Matemática, ambos da Universidade Federal do Paraná (UFPR). Paraná, Brasil. D orcid.org/0000-0001-8572-3758 $\bowtie$ artgoes@ufpr.br

Recebido em 10/06/2020 Aceito em 14/08/2020 Publicado em 28/09/2020 knowledge, not only of mathematical concepts, but also in an interdisciplinary approach.

Keywords: Teaching and learning. Problem solving. Active methodologies. Trends in Mathematics Education. 


\section{Introdução}

Os conteúdos da disciplina de Matemática são considerados por muitos estudantes como de difícil entendimento. Tal fato pode estar relacionado a algumas metodologias utilizadas por professores, em que se faz uso de recursos e métodos que não mostram a aplicabilidade dos conceitos matemáticos no cotidiano dos estudantes. As presentes estratégias consideram os estudantes apenas como ouvintes e receptores de informações, induzindo ao processo de memorização do conteúdo. Essas metodologias se aproximam da educação bancária, amplamente discutida por Paulo Freire (2016), em que, sobretudo, o aprender está fixo nos modos de resoluções e nas regras apresentadas pelo professor, sem oportunizar práticas pedagógicas diferenciadas trazendo significado para os conceitos/conteúdos científicos/escolares.

A vida da maioria dos estudantes, na atualidade, é permeada por informações proporcionadas por diversos aparatos tecnológicos. Assim, no que diz respeito à aprendizagem da Matemática, se faz cada vez mais necessária a utilização, por parte do professor, de metodologias que aproximem a realidade vivida pelos estudantes aos conceitos matemáticos, e também envolvam as outras áreas do conhecimento, por meio de abordagem interdisciplinar, compreendida como

interação existente entre duas ou mais disciplinas. Essa interação pode ir da simples comunicação de ideias à integração mútua dos conceitos diretores da epistemologia, da terminologia, da metodologia, dos procedimentos, dos dados e da organização referentes ao ensino e à pesquisa. (FAZENDA, 2011, p. 54)

É preciso que os professores façam uso das metodologias e tendências da Educação Matemática, pois estas, no entender de Góes e Góes (2015), possuem o objetivo de auxiliar 0 professor, proporcionando não só o interesse dos estudantes pela Matemática, mas também explorando suas ideias e potencialidades.

Dentre as tendências na Educação Matemática, pode-se citar a História da Matemática; a Resolução de Problemas; as Atividades Investigativas; a Etnomatemática; a Modelagem Matemática; e as Tecnologias Educacionais para o ensino e aprendizagem em Matemática (GÓES e GÓES, 2015). A Modelagem Matemática, tendência que se apresenta neste artigo, permite ao professor explorar conteúdos e conceitos matemáticos na resolução de problemas. Na Modelagem Matemática, apesar de ser possível prever conteúdos que serão abordados durante a atividade, outros conteúdos e conceitos matemáticos que não estavam no planejamento podem surgir, e, assim, "vale lembrar que a modelagem matemática não é presa a um currículo, tampouco há uma 
'ordem' para trabalhar conteúdos" (GÓES e GÓES, 2015, p. 115). Neste contexto, o presente estudo vislumbra uma aproximação da Modelagem Matemática com a metodologia STEAM (do inglês Science, Technology, Engineering, Arts \& Design and Mathematics, ou seja, Ciência, Tecnologia, Engenharia, Artes \& Design e Matemática, tradução nossa), para auxiliar na resolução de problemas do cotidiano.

Na compreensão de Lorenzin, Assumpção e Bizerra (2018), o STEAM é baseado em projetos que partem de problemas reais, com princípios possíveis de serem desenvolvidos no ambiente escolar, como a integração de conteúdos contextualizados das diferentes áreas de ensino, favorecendo o protagonismo do estudante na construção de seu conhecimento.

Desta forma, o presente estudo procura indicar as proximidades e convergências entre a Modelagem Matemática e o STEAM, de modo que o leitor possa identificar formas de proporcionar a aprendizagem e o conhecimento matemático aos estudantes, por meio de práticas diferenciadas na resolução de problemas no ambiente escolar.

Observa-se a importância da discussão realizada neste texto, uma vez que, na busca de pesquisas acadêmicas realizada no Catálogo de Teses e Dissertações da Coordenação de Aperfeiçoamento de Pessoal de Nível Superior (CAPES), não foram identificadas pesquisas com menção às duas metodologias concomitantemente. No levantamento bibliográfico realizado em abril de 2020, os descritores utilizados na referida base foram "educação", "STEAM" e "Modelagem Matemática", associados da seguinte maneira: educação e STEAM, com 28 pesquisas retornadas pela base de dados; Modelagem Matemática e STEAM, sendo indicadas 18 pesquisas; e Modelagem Matemática, STEAM e educação, em que não houve pesquisa retornada pela base de dados. Cabe ressaltar que o descritor STEAM foi associado à "educação" na primeira busca, pois essa metodologia é utilizada também em outras áreas do conhecimento.

Ao realizar a leitura dos títulos e resumos das 46 pesquisas não foi identificada discussão relacionada ao tema do presente estudo, o que pode indicar que os campos de estudos Educação Matemática - local onde a Modelagem Matemática está inserida - e Metodologias Ativas — local onde o STEAM está inserido — não apresentam conversas efetivas.

O presente estudo apresenta resultado inicial de discussões teóricas de uma pesquisa de mestrado profissional em desenvolvimento no Programa de Pós-Graduação em Educação: Teoria e Prática de Ensino da Universidade Federal do Paraná, cujo objetivo é analisar a prática do professor-pesquisador (primeiro autor deste texto) na abordagem de conceitos matemáticos no 


\section{Ensino Fundamental.}

Dessa forma, este texto está organizado em seis seções, sendo que na próxima é apresentada a Modelagem Matemática. Na seção 3 é apresentada a metodologia STEAM. Na próxima seção 4 apresentam-se as proximidades e convergências entre as duas metodologias indicadas. Na sequência, relata-se experiência docente por meio da Modelagem Matemática e STEAM. Por fim, são realizadas as discussões finais referentes à temática apresentada.

\section{Modelagem Matemática}

A Modelagem Matemática é uma metodologia que proporciona ao professor auxílio nas práticas pedagógicas aplicadas no âmbito escolar, a qual pode associar os conteúdos escolares com a realidade do estudante, não envolvendo exclusivamente a Matemática, mas também outras áreas do conhecimento. Segundo o documento da Base Nacional Comum Curricular (BNCC),

os processos matemáticos de resolução de problemas, de investigação, de desenvolvimento de projetos e da modelagem podem ser citados como formas privilegiadas da atividade matemática, motivo pelo qual são, ao mesmo tempo, objeto e estratégia para a aprendizagem ao longo de todo o Ensino Fundamental. (BRASIL, 2017, p. 267)

Na compreensão de Góes e Góes (2015), a Modelagem Matemática aplicada no contexto escolar busca mostrar ao estudante a importância da Matemática na resolução de problemas em diversas situações-problema presentes no cotidiano, pois proporciona a construção do conhecimento, do raciocínio lógico, além de motivação, autonomia e pensamento crítico. Com isso, o conceito de Modelagem Matemática, por Burak (1992, p. 62), "constitui-se em um conjunto de procedimentos cujo objetivo é construir um paralelo para tentar explicar, matematicamente, os fenômenos presentes no cotidiano do ser humano, ajudando fazer predições e a tomar decisões".

A Modelagem Matemática na Educação Básica, segundo Góes e Góes (2016, p. 49) tem como objetivo "aplicar ou representar conceitos, possibilitando aos estudantes a percepção da importância que a matemática tem no cotidiano deles. Dessa forma, o estudante pode ser motivado a desenvolver o raciocínio lógico e a ter uma visão crítica dos acontecimentos".

Segundo Burak (2016), a Modelagem Matemática vem sendo utilizada nas pesquisas brasileiras de pós-graduação, artigos, revistas entre outras, desde a época de 1980, com a intenção de apresentar a importância da Matemática no cotidiano. Assim, 
a modelagem matemática vem sendo estudada por pesquisadores que perceberam que, com avanço das tecnologias, muitas atividades passaram a ser realizadas pelas máquinas. Como consequência desse avanço, os conceitos matemáticos passam pelo cotidiano sem serem notados pela população que, em sua maioria, acredita que a matemática é utilizada somente nos bancos escolares. (GÓES e GÓES, 2016, p. 44)

As transformações - culturais, tecnológicas, relações sociais, entre outras - da sociedade exercem influência no cotidiano escolar, levando à necessidade de mudança na forma de ensinar e aprender. A escola, por sua vez, tentando atender a essa nova demanda, se esforça em desenvolver mudanças nas práticas pedagógicas. Sendo assim, professores se empenham em desenvolver estratégias pedagógicas que possibilitem maior integração de fenômenos do cotidiano à disciplina escolar.

Com a Matemática não é diferente, pois procura se adaptar para atender as exigências de uma sociedade que requer, cada vez mais, sujeitos ativos que busquem soluções para os problemas da realidade. Nessa disciplina escolar, por exemplo, é possível inserir estratégias que envolvam desenhos, imagens, gráficos, entre outras formas de representação, comuns no dia a dia. Essas representações podem ser denominadas de modelos, ao considerar que "um modelo é um conjunto de símbolos os quais interagem entre si representando alguma coisa" (BIEMBENGUT, 2004, p. 16).

Geralmente, na Modelagem Matemática, o estudante utiliza símbolos matemáticos para resolução de problema, apresentando expressões que, diante disso, recebem o nome de modelos matemáticos. Assim, "os modelos matemáticos procuram representar a realidade por meio de equações; no entanto, a resolução de equações nem sempre é de fácil entendimento, mesmo quando são usadas para representar um único fenômeno" (GÓES e GÓES, 2016, p. 14).

O vocábulo "modelo" tem alguns significados em diferentes áreas do conhecimento. De acordo com o Dicionário Houaiss da língua portuguesa (HOUAISS e VILLAR, 2009, p. 1304), a palavra modelo tem a definição: "1. representação, em escala reduzida de objeto, obra de arquitetura etc. a ser produzida em dimensões normais. [...] 4. reprodução tridimensional, ampliada ou reduzida de qualquer coisa real, us. como recurso didático".

Para Góes e Góes (2016, p. 14), "o objetivo de um modelo é que seja construído de tal forma que se possa entender a realidade de um modo mais simples e, ao mesmo tempo, o mais completo e preciso possível". Dessa forma, percebe-se que os conceitos sobre a palavra modelo, procuram facilitar o entendimento e funcionamento dos fenômenos do cotidiano para que os indivíduos possam interpretar cada detalhe do objeto construído. 
No ambiente escolar, para auxiliar o estudante em seus processos de aprendizagem, para que possa assimilar o conteúdo e o contextualizar à realidade, busca-se um apoio com outros tipos de modelos, um deles seria o modelo físico com a produção de um protótipo como casa, carros, usinas, entre outros, utilizados em diversas áreas do conhecimento, assim como na Matemática (GÓES e GÓES, 2016). Dessa forma, também pode ser compreendido o modelo matemático, não somente os elaborados por meio de equações algébricas, mas os protótipos que estão repletos de formas geométricas, considerando a Geometria como parte da Matemática, conforme a indicação nos documentos oficiais da educação como os Parâmetros Curriculares Nacionais PCN (BRASIL, 1998) e Base Nacional Comum Curricular — BNCC (BRASIL, 2017).

Para construção de um modelo físico, que no ambiente escolar é comumente denominado de maquete, mesmo não contando com o rigor de normas pertinentes à construção de tal objeto (GÓES e GÓES, 2016), podem ser utilizados diversos recursos, desde materiais recicláveis até a tecnologia de impressão 3d. Com isso, é possível utilizar as contribuições da Expressão Gráfica no ensino da Matemática. A saber, a

\footnotetext{
Expressão Gráfica é um campo de estudo que utiliza elementos de desenho, imagens, modelos, materiais manipuláveis e recursos computacionais aplicados às diversas áreas do conhecimento, com a finalidade de apresentar, representar, exemplificar, aplicar, analisar, formalizar e visualizar conceitos. Dessa forma, a expressão gráfica pode auxiliar na solução de problemas, na transmissão de ideias, de concepções e de pontos de vista relacionados a tais conceitos. (GÓES, 2013, p. 20)
}

Assim "podemos ainda ter sólidos geométricos e outros objetos, que são modelos físicos e ajudam na compreensão de conceitos escolares e científicos" (GÓES e GÓES, 2016, p. 16), proporcionando ao estudante a aprendizagem por meio de situações concretas com o auxílio das formas associadas à realidade.

Com a compreensão do conceito de modelo e alguns recursos para sua constituição, são apresentadas as etapas da Modelagem Matemática, que para Burak $(2004,2017)$ e Burak e Aragão (2012) se dividem em cinco etapas:

- escolha do tema - pode ser definido pelo interesse do grupo (estudantes e professor). 0 professor pode propor um tema e, ao mesmo tempo, mediar os interesses dos estudantes.

- pesquisa exploratória - busca de informações por meio dos recursos como internet, revistas, jornais, vídeos, livros, redes sociais, entrevistas entre outros.

- levantamento dos problemas - por meio da pesquisa exploratória, associar o tema aos 
conteúdos matemáticos e outras áreas do conhecimento.

- resolução de problemas e desenvolvimento do conteúdo matemático no contexto do tema - desenvolver estratégias para a resolução dos problemas coletados na etapa anterior, proporcionando a construção de modelos matemáticos ou físicos que possam desenvolver o interesse dos estudantes em verificar sua realidade.

- análise crítica das soluções - discutir e compreender as soluções encontradas por meio do aspecto matemático e outras áreas do conhecimento para construir o conhecimento associado com a realidade.

A visão da Modelagem Matemática por outros autores como Biembengut (2004), Góes e Góes (2016), Biembengut e Hein (2019) consiste em três etapas que são elas: interação, matematização e modelo matemático. Embora cada etapa se subdivida em subetapas como reconhecimento das situações problema, familiarização com assunto a ser modelado, reconhecimento das situações-problema, resolução do problema em termos do modelo, interpretação da solução e validação do problema. Essa forma de definir as etapas da Modelagem Matemática mostra a dinamicidade dessa metodologia, pois como destaca Góes e Góes (2016, p. 54) "a modelagem matemática pode ser considerada de forma dinâmica e não linear, ou seja, sempre que necessário, pode-se retornar qualquer uma das etapas durante o processo".

No entanto, como se pretende discutir as proximidades e convergência da Modelagem Matemática com a metodologia STEAM para resolução de problemas, serão utilizadas neste texto as cinco etapas sugeridas por Burak $(2004,2017)$ e Burak e Aragão (2012). Diante disso, considerando o entendimento dos autores citados, pode-se representar por meio da Figura 10 processo contínuo sobre Modelagem Matemática.

Observa-se na Figura 1 que a primeira etapa é a escolha do tema, sugerido pelo interesse do grupo, partindo de sua realidade, sendo que o tema pode ser indicado pelo professor ou definido conjuntamente com os estudantes, conforme seus interesses. No intermédio entre as duas etapas — escolha do tema e pesquisa exploratória - busca-se uma definição do tema, pelo estudante, 0 qual pode envolver, ou não, outras áreas do conhecimento. Dessa forma, a etapa da pesquisa exploratória, busca envolver os recursos como internet, revistas, artigos, vídeos, redes sociais entre outros, possibilitando ao estudante usá-los na busca de informações referentes a um tema, contribuindo na construção do conhecimento. 


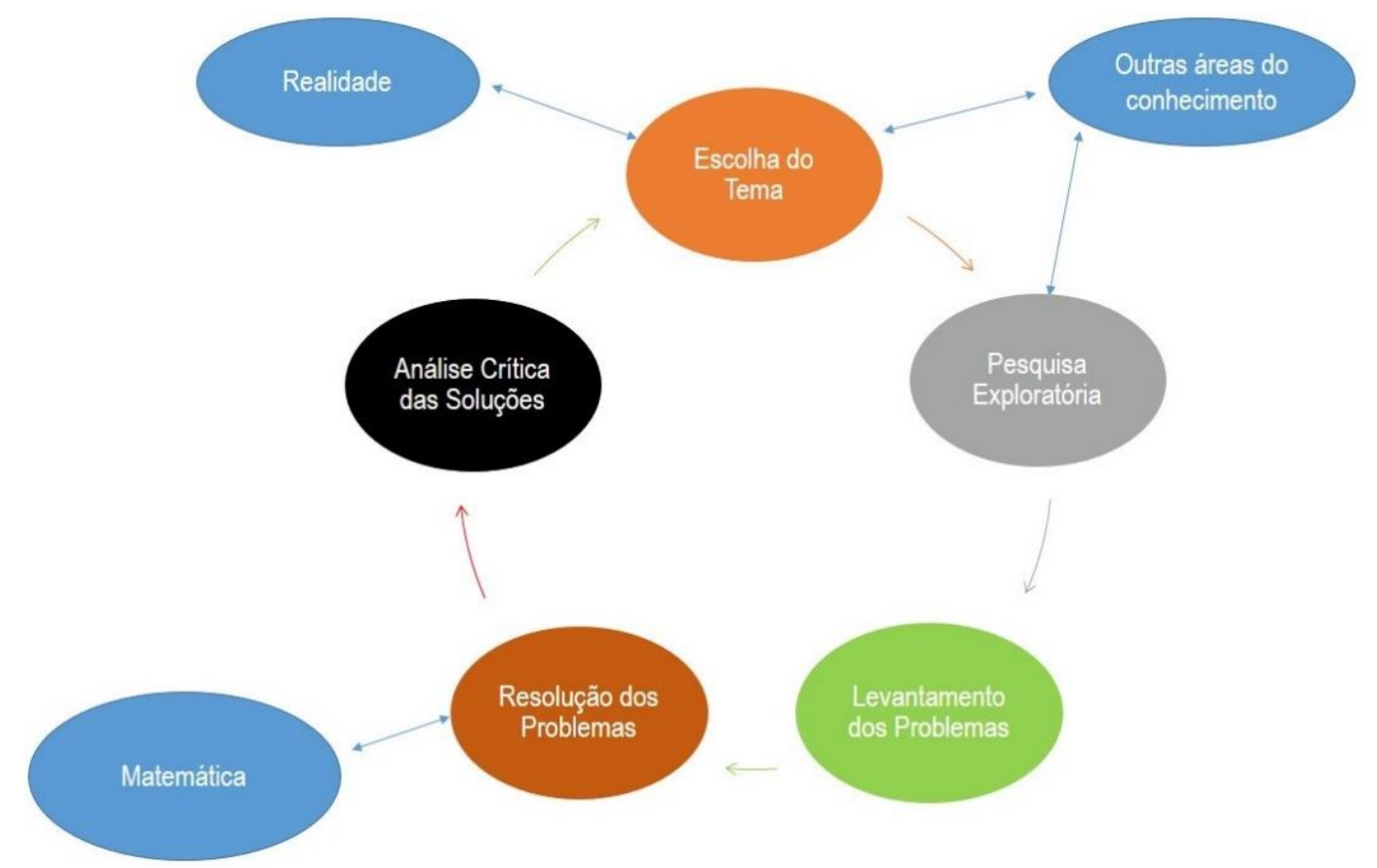

Figura 1: Processo da Modelagem Matemática (Adaptado de Burak, 2004; e Burak e Aragão, 2012)

O próximo passo é o levantamento dos problemas, momento no qual os estudantes devem coletar dados relevantes que possam proporcionar a compreensão de problemas que estejam associadas à realidade. Em seguida, os conceitos, dentre eles, os matemáticos, auxiliam a resolução dos problemas propostos na etapa anterior. E por fim, acontece a etapa final da Modelagem Matemática composta pela análise e pelas críticas, proporcionando ao estudante discussões e compreensões das apresentações dos modelos representados, contribuindo com os processos de ensino e aprendizagem dos estudantes envolvidos no desenvolvimento das etapas da Modelagem Matemática.

O processo apresentado sobre a Modelagem Matemática, possibilita a construção de modelos físicos e, desse modo, observa-se proximidades e convergências com a metodologia STEAM.

\section{Resolução de problemas por meio da metodologia STEAM}

O STEAM teve sua origem com o acrônico SMET, criado no início dos anos 1990 pela National Science Foundation (NSF) dos Estados Unidos. No entanto, no ano de 2001, o consenso da diretoria da NSF, decidiu-se por utilizar a sigla STEM, sendo espalhada em diversos países pela observância dos Estados Unidos em relação à carência nas profissões que envolvem as quatro áreas de conhecimento que a sigla STEM faz referência: Ciências, Tecnologias, Engenharia 
e Matemática (LORENZIN, ASSUMPÇÃO e BIZERRA, 2018). Mas, em virtude da baixa adesão pela metodologia STEM, no ano de 2008 é adicionado ao acrônico a letra "A", integrando a área de Artes \& Design, formando a sigla STEAM.

A inclusão de uma nova área abre a possibilidade de ampliar abordagem interdisciplinar com a Ciência, a Tecnologia, a Engenharia, a Artes \& Design e a Matemática, pois "a incorporação de Artes \& Design ao STEM contribui para um maior engajamento de alunos e professores, estimulando a criatividade e intensificando o desenvolvimento cognitivo, emocional e psicomotor, além de ampliar a visão para a compreensão do mundo" (LORENZIN, ASSUMPÇÃO e BIZERRA, 2018, p. 204).

Nesse contexto, o STEAM tem a finalidade de proporcionar melhoria ao ensino e buscar o desenvolvimento do estudante por carreiras científicas e tecnológicas nessas áreas. Ainda, essa abordagem interdisciplinar pode proporcionar ao estudante a associação de seus interesses em resolver problemas da realidade, integrando e buscando o conhecimento nas diversas áreas. Cabe ressaltar que não há necessidade de que as cinco áreas de conhecimento sejam utilizadas/necessárias para a resolução do problema apresentado.

A metodologia STEAM proporciona ao estudante a autonomia para explorar, observar e interpretar a resolução de problemas da realidade em seu processo de ensino e aprendizagem (VASQUEZ, SCHEIDER e COMER, 2013), possibilitando o desenvolvimento de habilidades de interpretação, de comunicação, de análise e de sínteses para construir e aplicar novos conhecimentos, associados as diversas tecnologias (LORENZIN, ASSUMPÇÃO e BIZERRA, 2018), sobretudo as atuais, como impressão $3 d$ para a construção de protótipos.

A integração das áreas do conhecimento é possível e necessária no ambiente escolar, uma vez que as escolas devem evoluir e transformar suas metodologias de ensino conforme a necessidade da sociedade (RILEY, 2012), que atualmente demanda por sujeitos criativos e colaborativos, habilidades, necessárias para carreiras do século XXI. Por meio do STEAM, há o aprofundamento de temas que contribuem com a formação do conhecimento, na medida em que podem proporcionar criação, reflexão, análise e investigação de soluções, por meio da resolução de problemas em seu contexto globalizado (ENGLISH, 2017).

No STEAM, o tema é proposto a partir do interesse do estudante, com a mediação do professor, em prol da interdisciplinaridade entre as áreas do conhecimento, desenvolvendo 0 processo de descoberta de como fazer algo para exercer a colaboração de aprendizagem com 0 
coletivo. Definida a temática a ser estudada, a metodologia STEAM, segundo Garofalo (2019) se atém a cinco etapas básicas para desenvolver o processo de incentivar 0 estudante nas descobertas, conforme Figura 2.

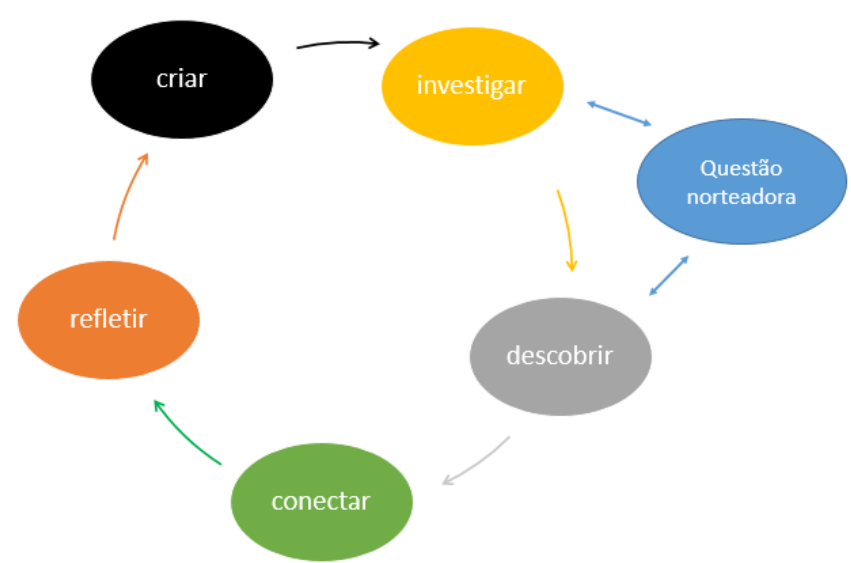

Figura 2: Processo das cinco etapas básica do STEAM (Adaptado de Garofalo, 2019)

Após a definição da temática a ser estudada, a primeira etapa contempla o investigar, a qual possibilita uma questão norteadora do interesse do estudante, sendo um tema da realidade, advindo de revistas, jornais, artigos, internet, redes sociais, entrevistas entre outros. Esta ação proporciona ao estudante a pesquisa para descobrir e aprofundar conceitos do tema da realidade proposta. A partir das possibilidades de envolvimento das diversas áreas do conhecimento e, diante das discussões, estudos e apropriação de conceitos acerca do tema, o estudante pode conectá-los para resolução de problemas do cotidiano por meio da investigação. Isso favorece a leitura, codificação e interpretação dos dados e informações obtidos durante a pesquisa. Em seguida acontece o refletir, que proporciona ao estudante a análise - com o grupo de trabalho das hipóteses de construção, decorrentes do processo de resolução de problemas. Finalmente, torna-se possível o criar, etapa na qual acontece a solução propriamente dita. Pode-se citar um exemplo que ilustra esta etapa final: a criação de um protótipo com utilização de produtos alternativos, impressora 3d, entre outros modelos, enquanto solução do problema.

Observa-se que as etapas do STEAM, segundo Lorenzin, Assumpção e Bizerra (2018) e Bacich e Holanda (2020), possuem processo semelhante a aprendizagem baseada em projetos: questão norteadora, pesquisa, levantamento de ideias e produto final, permitindo integrar disciplinas de diferentes áreas do conhecimento e seus diversos conteúdos, fazendo com que 0 estudante seja protagonista da aprendizagem, descobrindo e criando soluções para resolução de problemas que 
precisam estar ancorados [os problemas] em temas que façam parte da realidade dos estudantes, seja no próprio ambiente escolar, seja em sua moradia, seja em sua comunidade, permitindo também uma aproximação dos conceitos que são abordados na escola com os problemas reais do cotidiano dos estudantes. (BACICH e HOLANDA, 2020, p. 31)

Desse modo, as etapas da metodologia STEAM podem proporcionar ao estudante experiências vivenciadas nas resoluções de problemas que envolvem investigação e compreensão do pensamento científico, crítico e criativo, possibilitando, também, a ludicidade, em todos os níveis de ensino, desde a Educação Infantil ao Ensino Superior. Ainda, Cuginotti (2020, p. 229) afirma que "as reflexões sobre as conexões entre elas [disciplinas STEAM] envolvem pensamento crítico e habilidades de resolução de problemas que a abordagem STEAM em sala de aula é capaz de fomentar, impactando, dessa forma, positivamente a aprendizagem dos estudantes".

Apesar de não ser citada na Base Nacional Comum Curricular (BNCC), a concepção do STEAM está inserida de forma indireta neste documento, mostrando que sua efetivação é respaldada, principalmente, ao auxiliar os professores no cumprimento das competências gerais de ensino:

Exercitar a curiosidade intelectual e recorrer à abordagem própria das ciências, incluindo a investigação, a reflexão, a análise crítica, a imaginação e a criatividade, para investigar causas, elaborar e testar hipóteses, formular e resolver problemas e criar soluções (inclusive tecnológicas) com base nos conhecimentos das diferentes áreas. (BRASIL, 2017, p. 9, grifos nossos)

Também, ao analisar competências gerais da BNCC é possível intensificar a assimilação com a metodologia STEAM, conforme os recortes a seguir:

Utilizar diferentes linguagens - verbal (oral ou visual-motora, como Libras, e escrita), corporal, visual, sonora e digital - , bem como conhecimentos das linguagens artística, matemática e científica, para se expressar e partilhar informações, experiências, ideias e sentimentos em diferentes contextos e produzir sentidos que levem ao entendimento mútuo.

Compreender, utilizar e criar tecnologias digitais de informação e comunicação de forma crítica, significativa, reflexiva e ética nas diversas práticas sociais (incluindo as escolares) para se comunicar, acessar e disseminar informações, produzir conhecimentos, resolver problemas e exercer protagonismo e autoria na vida pessoal e coletiva.

Valorizar a diversidade de saberes e vivências culturais e apropriar-se de conhecimentos e experiências que the possibilitem entender as relações próprias do mundo do trabalho e fazer escolhas alinhadas ao exercício da cidadania e ao seu projeto de vida, com liberdade, autonomia, consciência crítica e responsabilidade. 
Argumentar com base em fatos, dados e informações confiáveis, para formular, negociar e defender ideias, pontos de vista e decisões comuns que respeitem e promovam os direitos humanos, a consciência socioambiental e o consumo responsável em âmbito local, regional e global, com posicionamento ético em relação ao cuidado de si mesmo, dos outros e do planeta.

Agir pessoal e coletivamente com autonomia, responsabilidade, flexibilidade, resiliência e determinação, tomando decisões com base em princípios éticos, democráticos, inclusivos, sustentáveis e solidários. (BRASIL, 2017, p. 9-10, grifos nossos)

Os indicativos da BNCC são aspectos que o STEAM contempla e favorecem ao estudante a aprendizagem contextualizada com a realidade, proporcionando a integração de outras áreas do conhecimento que possam auxiliar na resolução de problemas, contribuindo para a formação integral do estudante, na qual ele é o protagonista de todo processo. Com isso, pode desenvolver a autonomia na criação, na elaboração, na testagem de soluções para resolução de problemas, aprofundando conceitos e identificando dados relevantes que possam contribuir com a criação do produto final.

Tendo sido contemplados nesta seção a apresentação do termo STEM e o esclarecimento a respeito da sua origem e ampliação para STEAM, com a finalidade de possibilitar uma abordagem interdisciplinar da metodologia e, principalmente, considerando que pode ser baseada em projetos, verifica-se que pode proporcionar ao estudante a autonomia para explorar, observar e interpretar a resolução de problemas da realidade em seu processo de ensino e aprendizagem, fatos que a aproximam da Modelagem Matemática em muitos aspectos. Sendo assim, na próxima seção serão apresentadas as proximidades e convergências entre as duas metodologias: Modelagem Matemática e STEAM.

\section{Modelagem Matemática e STEAM: proximidades e convergências}

Ao observar as tendências educacionais para Matemática é possível verificar que a Modelagem Matemática pode contribuir para os processos de ensino e de aprendizagem, na medida em que contempla a realidade dos estudantes e pode integrar diferentes áreas de conhecimento. Considerando o explicitado nas seções anteriores, a Modelagem Matemática pode ter como produto final (modelo) a elaboração de modelos físicos, com isso, vê-se a aproximação com a metodologia STEAM, uma vez que ambas as metodologias possibilitam a resolução de problemas do cotidiano dos estudantes, bem como a construção do conhecimento e a busca de possiveis soluções. 
Dessa forma, o Quadro 1 apresenta proximidades e convergências entre a Modelagem Matemática e o STEAM, associando as etapas que se complementam e compõe cada uma das metodologias. Cabe ressaltar que a teoria do STEAM não apresenta a escolha do tema como uma das etapas, no entanto, ela é anterior as cinco etapas apresentadas no Quadro 1.

Quadro 1: Proximidades entre as duas metodologias

\begin{tabular}{|c|c|c|c|c|}
\hline \multicolumn{2}{|c|}{ Modelagem Matemática } & \multicolumn{3}{|c|}{ STEAM } \\
\hline Etapa & Ações & Etapa & & Ações \\
\hline \multicolumn{2}{|c|}{ Escolha do tema $\rightarrow$ Interesse do estudante } & \multirow{2}{*}{ Investigar } & \multirow{2}{*}{$\rightarrow$} & \multirow{2}{*}{$\begin{array}{l}\text { Questão norteadora } \\
\text { do Tema }\end{array}$} \\
\hline \multirow{2}{*}{\multicolumn{2}{|c|}{ Pesquisa exploratória $\rightarrow \begin{array}{l}\text { Aprofundamento do } \\
\text { tema }\end{array}$}} & & & \\
\hline & & \multirow{2}{*}{ Descobrir } & \multirow{2}{*}{$\rightarrow$} & \multirow{2}{*}{$\begin{array}{l}\text { Identificação do } \\
\text { Problema }\end{array}$} \\
\hline \multirow{2}{*}{\multicolumn{2}{|c|}{$\begin{aligned} \text { Levantamento dos } \\
\text { problemas }\end{aligned} \rightarrow \begin{array}{l}\text { problemas por meio } \\
\text { da coleta de dados da } \\
\text { pesquisa }\end{array}$}} & & & \\
\hline & & \multirow[t]{2}{*}{ Conectar } & \multirow[t]{2}{*}{$\rightarrow$} & \multirow[t]{2}{*}{ Geração de ideias } \\
\hline \multirow{2}{*}{$\begin{array}{r}\text { Resolução de } \\
\text { problemas e } \\
\text { desenvolvimento do } \\
\text { conteúdo matemático } \\
\text { no contexto do tema }\end{array}$} & \multirow[b]{2}{*}{$\rightarrow \begin{array}{l}\text { Construção de } \\
\text { modelos matemáticos }\end{array}$} & & & \\
\hline & & \multirow[t]{2}{*}{ Refletir } & \multirow[t]{2}{*}{$\rightarrow$} & \multirow[t]{2}{*}{ Desenvolvimento } \\
\hline \multirow{2}{*}{$\begin{array}{r}\text { Análise crítica das } \\
\text { soluções }\end{array}$} & \multirow{2}{*}{$\begin{aligned} & \text { Compreender e } \\
& \rightarrow \text { discutir as soluções } \\
& \text { encontradas }\end{aligned}$} & & & \\
\hline & & Criar & $\rightarrow$ & Produto Final \\
\hline
\end{tabular}

Fonte: Elaboração dos Autores

O Quadro 1 apresenta as relações existentes entre as fases da Modelagem Matemática e do STEAM, por exemplo, realizando a leitura do Quadro 1 da esquerda para a direita, na segunda fase da Modelagem Matemática "Pesquisa exploratória" há a conclusão da fase "Investigar" e inicia-se a fase "Descobrir" do STEAM. Cabe ressaltar que as relações também podem ser observadas ao realizar a leitura do Quadro 1 da direita para a esquerda. Esse é motivo do "desnível" apresentado no Quadro 1, visando indicar aproximações entre as etapas da Modelagem Matemática e do STEAM.

A Modelagem Matemática observa-se a escolha do tema, de acordo com o interesse do estudante, como primeira etapa. O STEAM também inicia seu processo de modo semelhante. No entanto, como dito anteriormente, este momento não é considerado como uma de suas etapas, 
mas após a escolha do tema, o STEAM investiga a temática, elaborando uma questão norteadora.

A definição de uma questão norteadora é essencial para o desenvolvimento de uma pesquisa exploratória, para que o tema seja aprofundado, momento este indicado como a segunda etapa da Modelagem Matemática. Também é nesse momento que a Modelagem Matemática identifica com maior clareza o problema a ser resolvido, visto que, por meio do aprofundamento da temática, o estudante terá maior familiaridade com o tema. Com isso, há clareza na identificação dos problemas, segunda fase do STEAM.

Com a identificação dos problemas, mediada pelo professor, a Modelagem Matemática proporciona a terceira etapa para que o estudante possa levantar e coletar dados desses problemas relacionados com sua realidade. É neste momento que o estudante gera ideias para solucionar os problemas que foram levantados, identificada como a terceira fase do STEAM.

Considerando as ideias que surgem, há a discussão sobre resolução de problemas e desenvolvimento do conteúdo matemático no contexto do tema, auxiliando as soluções por meio da construção de modelos matemáticos, quarta etapa da Modelagem Matemática. Assim observase que, nesta ocasião da quarta fase do STEAM, realiza-se desenvolvimento dessas ideias que podem proporcionar a reflexão e compreensão do estudante para soluções dos problemas. Cabe ressaltar que no STEAM, geralmente, se espera a interdisciplinaridade ao menos entre duas das áreas que definem tal sigla.

Por fim, a Modelagem Matemática apresenta a quinta etapa na qual são realizadas as análises e críticas das soluções encontradas, sendo um momento rico em discussões e apresentações das soluções que podem não envolver somente conteúdos matemáticos, mas também os de outras áreas do conhecimento. Com isso, é oportunizada ao estudante a criação de um produto final que é construído, materializado, no qual são apresentadas e testadas as soluções dos problemas, como propõe a quinta fase do STEAM.

Com essa análise do Quadro 1, é possível verificar as proximidades e convergências das duas metodologias. Ainda, retomando as discussões realizadas nas seções anteriores, é possível realizar levantamento de mais questões relevantes ao ensino da Matemática.

A etapa criar pode contribuir no desenvolvimento abstrato e lúdico de aprendizagem do estudante, possibilitando o desenvolvimento de sua autonomia e responsabilidade.

Modelagem Matemática e STEAM podem ser implantadas em qualquer nível de ensino, 
da Educação Infantil ao Ensino Superior. No entender de Góes e Góes (2016), a Modelagem Matemática proporciona ao estudante a resolução de situações-problema, associando os conteúdos escolares com sua realidade, já o STEAM considera que o estudante, por meio de um contexto globalizador, pode buscar soluções para resolver problemas da realidade $(\mathrm{BAClCH}$ e HOLANDA, 2020).

Desse modo, percebe-se que as metodologias fazem referência à realidade vivenciada pelo estudante e isto permite definir o tema ou questão norteadora dos conteúdos trabalhados, porém estes devem ser adequados ao estudante e ao período de ensino. Nesse sentido, proporciona ao estudante uma aprendizagem de forma significativa e colaborativa, pois possibilita a investigação do ensino da Matemática contextualizado com outras áreas do conhecimento.

As duas metodologias podem proporcionar práticas pedagógicas diferenciadas na forma de interpretação de textos, enunciados, gráficos, desenhos, tabelas entre outras, como também na formulação em diferentes modelos que podem ser matemáticos ou físicos, possibilitando por meio delas "valorizar e utilizar os conhecimentos historicamente construídos sobre o mundo físico, social, cultural e digital para entender e explicar a realidade, continuar aprendendo e colaborar para a construção de uma sociedade justa, democrática e inclusiva" (BRASIL, 2017, p. 09, grifos nossos).

Essas práticas pedagógicas podem ser desenvolvidas por meio da pesquisa, na qual 0 estudante utiliza recursos como: visitas, simuladores, jornais, revistas, artigos, livros, redes sociais, internet entre outros. Neste processo, o estudante investiga, sendo inserido no problema, no contexto da observação, na interpretação e na compreensão. Deste modo é possível realizar a coleta dos dados e informações sobre o tema, compreendendo que a pesquisa é

conhecer mais sobre o tema, ou seja, buscar informações no local onde se localiza o interesse do grupo de pessoas envolvidas, além de se construir em uma das premissas para o trabalho nessa visão de Modelagem, é uma etapa importante na formação de um estudante mais crítico, mais atento. (BURAK e ARAGÃO, 2012, p. 93)

Na metodologia STEAM, os estudantes discutem em grupos seus pontos de vista sobre os dados coletados acerca da pesquisa (CUGINOTTI, 2020). Na Modelagem Matemática os dados coletados por meio da pesquisa podem proporcionar a descoberta e a formulação de possibilidades para resolução de problemas a partir do cotidiano (GÓES e GÓES, 2016).

Ao analisar os dados coletados e as informações sobre o tema, o estudante pode articular 
o levantamento de possíveis subproblemas e propor formas de criar o modelo matemático, pois 0 estudante, com a mediação do professor, tem a capacidade de construir, levantar e propor seu próprio problema, advindo dos dados coletados (BURAK e ARAGÃO, 2012). Esta ação proporciona a conexão do levantamento dos problemas com o processo de sua resolução por meio do modelo matemático, ao mesmo tempo que, na metodologia STEAM, os grupos anotam todas as ideias possíveis e analisam as prováveis soluções do problema obtendo assim a sua reflexão da resolução dos problemas. Segundo Cuginotti (2020, p. 227), "nesse momento, os grupos devem olhar para tudo o que foi proposto, avaliar quais partes podem ser aproveitadas e o que deve ser deixado de lado momentaneamente".

Ao realizar a Modelagem Matemática por meio da resolução de problemas, o estudante utiliza seu conhecimento prévio em Matemática e, em razão disso, pode ocorrer a ausência de conhecimentos matemáticos que sejam pré-requisitos para a solução. Neste momento, o professor possui o papel fundamental de mediar a construção da aprendizagem (BURAK e ARAGÃO, 2012), destacando a importância de conteúdos matemáticos. Na metodologia STEAM, os grupos de trabalhos organizam as ideias que foram discutidas, sendo possível refletir em diversas áreas do conhecimento, de que forma irão organizar os conteúdos que precisam aprender e definir como colocar a ideia em prática. De acordo com Cuginotti (2020), cada grupo poderá escolher um caminho diferente para resolução do problema e, desta forma, destaca-se o fundamental papel do professor que realiza mediações necessárias em prol do processo de aprendizagem dos grupos.

Por fim, ocorrem as discussões das análises e das soluções apresentadas, oportunidade na qual os estudantes observam os processos de levantamentos dos problemas, em virtude das ponderações e das hipóteses (BURAK e ARAGÃO, 2012). No mesmo momento a metodologia STEAM possibilita ao estudante criar um produto final, sendo exemplo a construção de um protótipo, que não precisa ser em tamanho real, mas deve passar pelas etapas sugeridas: construção, apresentação e testes (CUGINOTTI, 2020). A construção de um protótipo pode ser desenvolvida em todas as etapas e tem a representação do eixo estruturante da Geometria, o qual pode ser utilizado para abordar conteúdos geométricos envolvendo diferentes situações do cotidiano.

A Figura 3 apresenta as proximidades e convergências entre as duas metodologias, conforme o texto apresentado. 


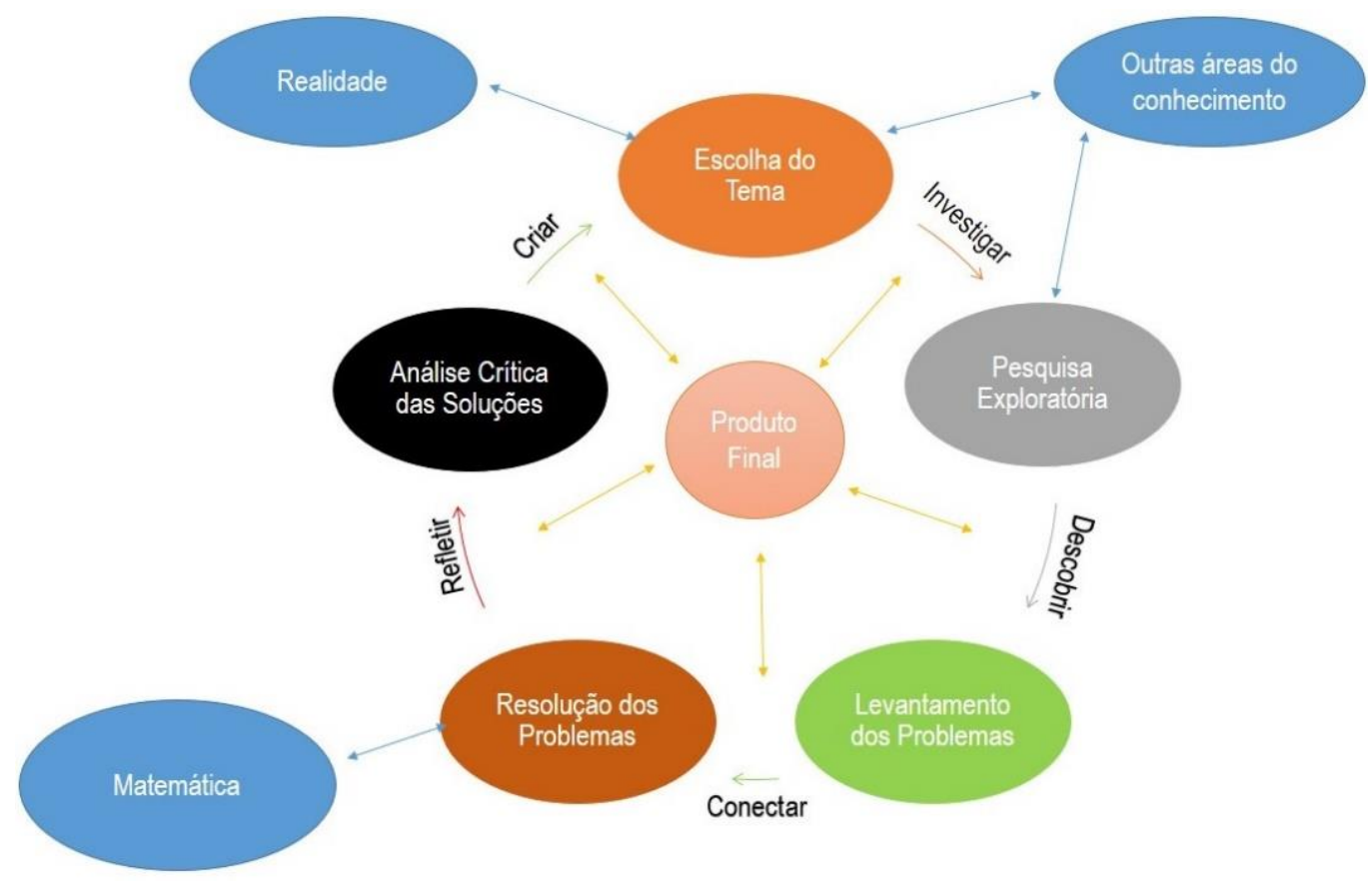

Figura 3: Processo entre as duas metodologias (Elaboração dos Autores)

Desse modo, apresentam-se as proximidades e convergências entre a Modelagem Matemática e a metodologia STEAM, propondo a junção das duas formas de ensino para que 0 estudante vivencie sua realidade, e possa tornar-se um cidadão crítico por meio de sua autonomia favorecida pelas discussões, reflexões e novas visões de sua aprendizagem (BURAK e ARAGÃO, 2012). Contudo, observa-se que a metodologia STEAM pode colaborar com a Modelagem e a resolução de problemas, contextualizando conteúdo matemático a outras áreas do conhecimento.

\section{Modelagem Matemática e STEAM em uma experiência docente}

Na descrição metodológica da pesquisa de Góes e Luz (2009), relacionada à construção de uma maquete da escola durante as aulas de Matemática, se evidenciam as presenças da Modelagem Matemática e do STEAM, ainda que os autores não façam uso de tais terminologias. Tais afirmações são possíveis de serem realizadas, visto que um dos autores do trabalho (GÓES e LUZ, 2009) é, também, pesquisador do presente estudo. Desta forma, ao revisitar suas pesquisas e tendo o discutido neste texto, as proximidades e convergências que podem ocorrer entre a Modelagem Matemática e o STEAM são destacadas a seguir, evidenciando as etapas indicadas na Figura 3 e Quadro 1.

Góes e Luz (2009) indicam que o tema abordado durante todo o ano letivo foi sugerido pelo professor e aceito pelos estudantes. Com isso, a investigação se inicia com a elaboração de 
esboço da planta baixa da escola, indicando todos os ambientes da instituição conhecidos/lembrados pelos estudantes. Com esse desenho ocorreu a pesquisa exploratória, visitando todos os ambientes da instituição e comparando as representações gráficas realizadas com o espaço físico real. Neste momento os participantes descobriram ambientes por eles desconhecidos, efetuando as devidas correções no esboço da planta baixa.

Com o novo desenho da planta baixa da escola, os estudantes levantaram o problema: como elaborar com precisão a planta baixa. Após as discussões e as diversas gerações de ideias para resolução concluíram: necessidade de obtenção das dimensões dos ambientes; elaboração do desenho em escala; utilização dos instrumentos de desenho geométrico. Para isso, os estudantes utilizaram trenas para medir todos os ambientes da escola e exploraram os conteúdos matemáticos referentes à escala, construções geométricas e transformações métricas, fundamentais para a desenvolvimento/elaboração da planta baixa, considerada aqui como um modelo matemático advindo do eixo norteador Geometria na disciplina de Matemática.

Com a planta baixa elaborada, os estudantes analisaram o construído até o momento e levantaram novo problema na continuação da elaboração da maquete da escola: determinação da altura das paredes e do telhado. Para isto, foram abordados conteúdos relacionados à semelhança de triângulo para o cálculo de altura inacessível, bem como, contextualizadas as formas geométricas tridimensionais e o cálculo de área e volumes.

Por fim, os estudantes desenharam as paredes e o telhado conforme as medidas já transformadas nas escalas trabalhadas, fizeram os recortes necessários em materiais determinados para a construção do protótipo/maquete. Góes e Luz (2009) trazem como resultado dessa prática a Figura 4, maquete de um dos blocos da escola.

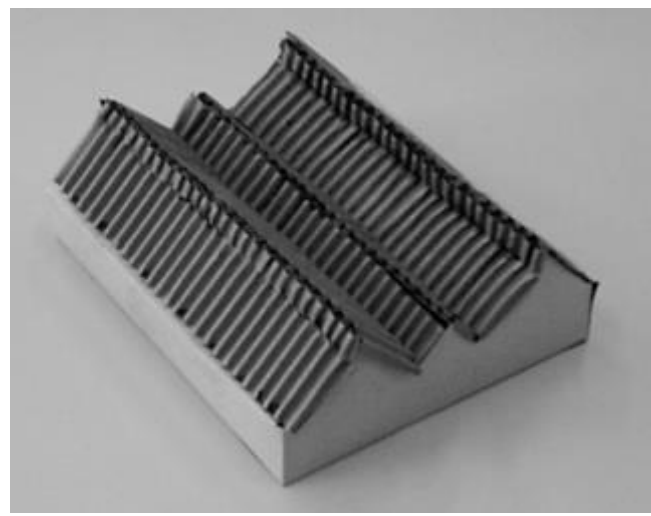

Figura 4: Maquete de um bloco da escola (GÓES e LUZ, 2009, p. 11)

A pesquisa de Góes e Luz (2009) contempla algumas das áreas do STEAM, como a 
Engenharia, Tecnologia, Artes e Matemática ao construir a maquete. Quanto à Tecnologia, a que pode ser menos evidente na descrição da experiência docente, se fez presente por meio de pesquisa na internet para consultar os materiais para realizar a maquete, bem como, os próprios materiais e ferramentas utilizados (KENSKI, 2012) como lápis, papel e materiais geométricos (compasso, esquadro, réguas, entre outros).

As etapas destacadas nessa subseção mostram as proximidades e convergências entre a Modelagem Matemática e o STEAM na pesquisa de Góes e Luz (2009) e, também, podem ser visualizadas nas Figuras 5 e 6.

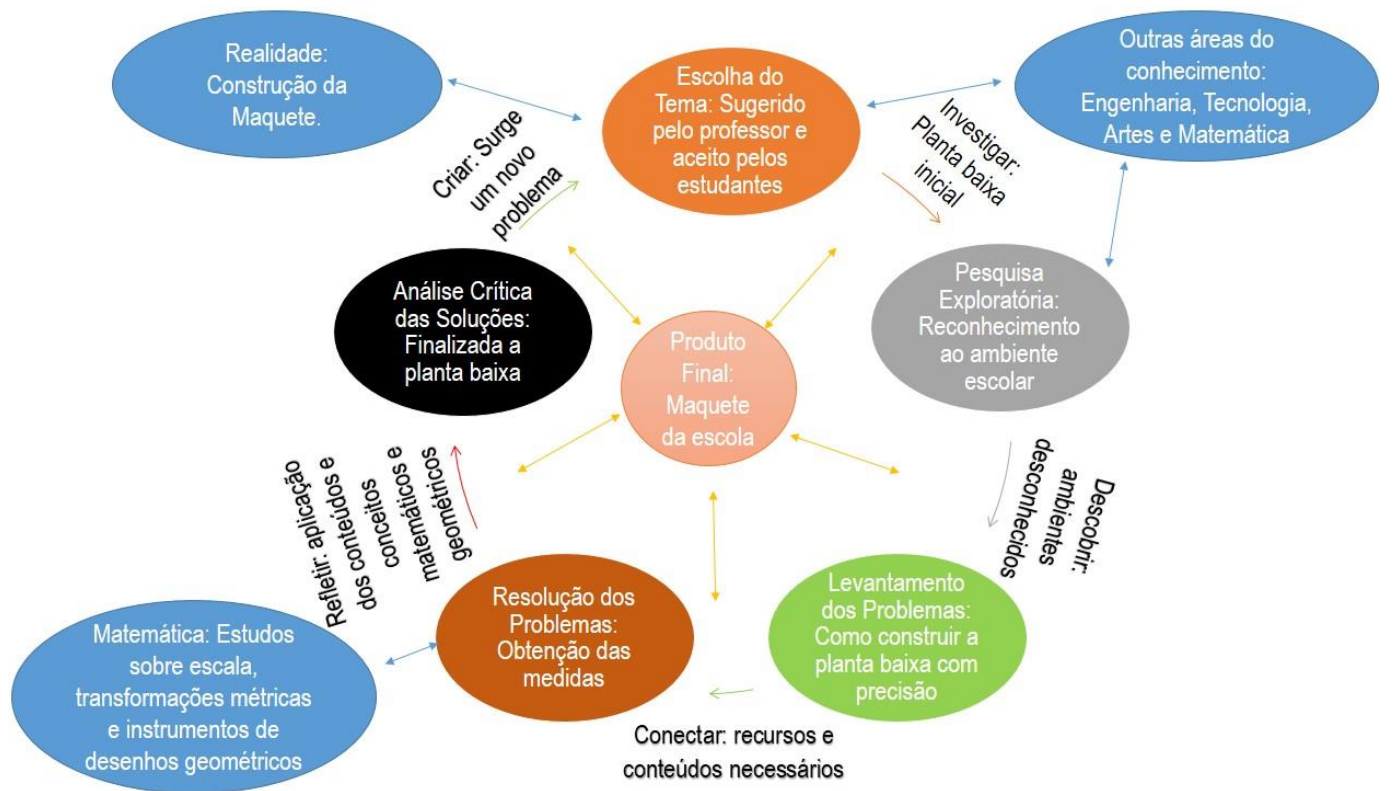

Figura 5: Elaboração da planta baixa - início da experiência didática (Elaborado pelo Autores)

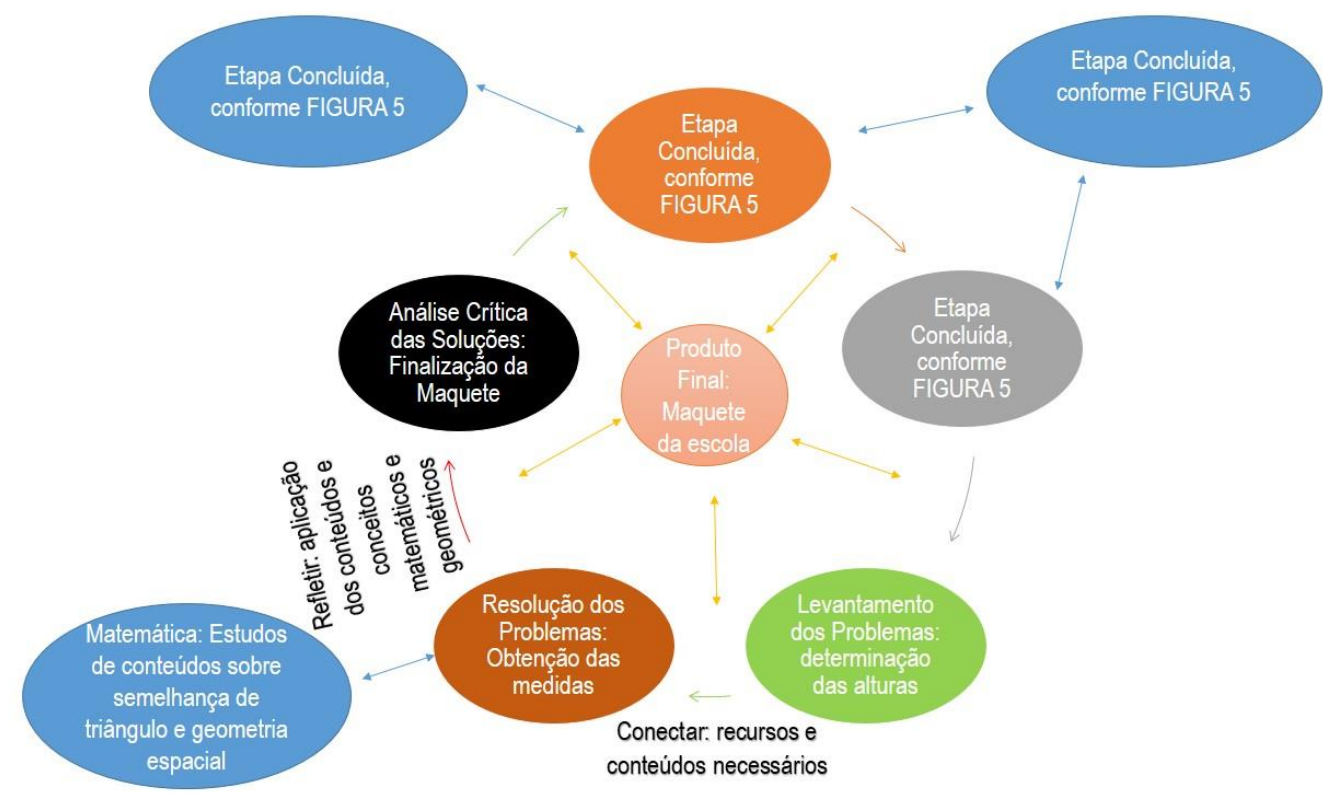

Figura 6: Elaboração da maquete - final da experiência didática (Elaborado pelo Autores) 
$\mathrm{Na}$ Figura 5 estão indicas as etapas desde a escolha do tema à construção da planta baixa quando surge novo problema que consistiu em determinar as alturas das paredes e telhado para finalizar a maquete, cuja etapas de resolução são apresentadas na Figura 6.

\section{Considerações Finais}

Metodologias de ensino que contribuam para que o estudante compreenda, interprete sua vivência/realidade, sendo capaz de transformá-la, relacionando os conteúdos escolares/científicos com diversas áreas de conhecimento é o que demandam os processos de ensino e aprendizagem, bem como a sociedade atual. Procurando evidenciar tais metodologias, o presente texto apresentou duas delas: uma específica do campo de estudos da Educação Matemática, a Modelagem Matemática; e a outra do campo de estudos das metodologias ativas, o STEAM.

A escolha de tais metodologias surge da ausência que parece haver no âmbito acadêmico, pois como descrito na introdução deste texto, não foram identificadas pesquisas que discutam, concomitantemente, a Modelagem Matemática e o STEAM.

Tanto a Modelagem Matemática quanto o STEAM são flexíveis em suas etapas e podem sofrer alterações e adaptações constantemente, sem perder o sentido de seus objetivos. $\mathrm{Na}$ Modelagem Matemática criam-se modelos matemáticos para resolução de problemas, o que possibilita a interdisciplinaridade, visto que os problemas estão relacionados às situações do contexto vivido pelos estudantes. Enquanto o STEAM apresenta características baseadas no desenvolvimento de projetos, permitindo a associação de duas ou mais áreas do conhecimento, porém sem a necessidade de contemplar todas em um mesmo projeto, resultando em produto final como, por exemplo, a construção de um protótipo, uma apresentação, uma campanha de conscientização entre outras.

As duas metodologias contribuem com a aprendizagem do estudante e tal fato é destacado em todo curso deste texto, ao evidenciar as práticas pedagógicas favorecidas pelo STEAM, que se encontram presentes no documento BNCC, bem como, a sugestão de utilização da metodologia de Modelagem Matemática na disciplina de Matemática, também por tal documento. Ainda, proporciona ao estudante por meio das metodologias citadas, a possibilidade de levantar dados, questionamentos, imaginar, planejar, criar, testar a resolução de um problema, abordando teorias, conceitos e cálculos da geometria, em abordagem interdisciplinar, considerando que o estudante pode ser o protagonista de sua aprendizagem, buscando 
informações, compartilhando ideias e estimulando sua criatividade, pois diante disso pode-se analisar suas dificuldades e facilidades de aprender no contexto de sua realidade.

Na Modelagem Matemática os modelos matemáticos "são construídos quando deseja-se expressar uma situação que enseja novos elementos ou alguma situação" (BURAK e ARAGÃO, 2012, p. 97). Esses modelos matemáticos, segundo Góes e Góes (2016), podem ser algébricos ou geométricos, representando de forma simplificada a realidade. No STEAM é previsto que o produto final seja algo construído pelos estudantes no desenvolver da temática (BACICH e HOLANDA, 2020). Ainda, no STEAM, "esse produto é geralmente um artefato, e sua produção poderá servir como base para o desenvolvimento de habilidades das diferentes áreas do conhecimento" (BACICH e HOLANDA, 2020, p. 38).

Concluindo, este estudo se ateve a indicar as proximidades e convergências entre a Modelagem Matemática e o STEAM ao analisar o processo de desenvolvimento e as etapas de cada uma delas, não evidenciando diferenças. Observa-se que não há necessidade que uma metodologia dependa da outra, mas a compreensão que essas metodologias possuem proximidades e convergências aproximando e entrelaçando os campos de estudos Educação Matemática e Metodologias Ativas, colaborando/auxiliando o professor e os estudantes a desenvolverem trabalhos que podem proporcionar aprendizagem, reflexão e autonomia na construção do conhecimento.

Dessa forma, como próximos passos desta pesquisa considera-se a importância de implementação de uma proposta pedagógica que considere a realidade dos estudantes mediadas pelo professor na resolução de problema a partir das duas metodologias apresentadas.

\section{Referências}

BACICH, Lilian; HOLANDA, Leandro. A aprendizagem baseada em projetos e a abordagem STEAM. In: BACICH, Lilian; HOLANDA, Leandro. (Org.). STEAM em sala de aula: aprendizagem baseada em projetos integrando conhecimento na Educação Básica. Porto Alegre: Penso, 2020, p. 29-50.

BIEMBENGUT, Maria Salett. Modelagem Matemática \& implicações no ensino e na aprendizagem matemática. Blumenau: EdFURB, 2004.

BIEMBENGUT, Maria Sallet; HEIN, Nelson. Modelagem matemática no ensino. São Paulo: Contexto, 2019. 
BRASIL. Ministério da Educação. Secretaria de Educação Básica. Base Nacional Comum Curricular. Brasilia: MEC/SEB, 2017.

BRASIL. Ministério da Educação. Secretaria de Educação Fundamental. Parâmetros Curriculares Nacionais: Matemática. Brasília: MEC/SEF, 1998.

BURAK, Dionísio. Modelagem Matemática e a sala de aula. In: ENCONTRO PARANANENSE DE MODELAGEM EM EDUCAÇÃO MATEMÁTICA, 1, 2004, Londrina. Anais do I EPMEM. Londrina: UEL, 2004, p. 1-10.

BURAK, Dionísio. Modelagem Matemática: ações e interações no processo de ensino e aprendizagem. 1992. 459f. Tese (Doutorado em Educação) — Faculdade de Educação. Universidade Estadual de Campinas. Campinas.

BURAK, Dionísio. Modelagem na perspectiva da Educação Matemática: um olhar sobre seus fundamentos. UNIÓN, v. 13, n. 51, p. 9-26, dez. 2017.

BURAK, Dionísio. Uma perspectiva de Modelagem Matemática para o ensino e a aprendizagem da Matemática. In: BRANDT, Celia Finck; BURAK, Dionísio; KLÜBER, Tiago Emanuel. (Org.). Modelagem Matemática: perspectivas, experiências, reflexões e teorizações. 2 ed. Ponta Grossa: EdUEPG, 2016, p. 17-40.

BURAK, Dionísio; ARAGÃO, Rosália Maria Ribeiro de. A modelagem matemática e relações com a aprendizagem significativa. Curitiba: CRV, 2012.

CUGINOTTI, Andressa Prata Cirino. STEAM na prática: exemplos de projetos. In: $\mathrm{BACICH}$, Lilian; HOLANDA, Leandro. (Org.). STEAM em sala de aula: aprendizagem baseada em projetos integrando conhecimento na Educação Básica. Porto Alegre: Penso, 2020, p. 213-229.

ENGLISH, Lyn D. Advancing elementary and middle school STEM Education. International Journal of Science and Mathematics Education, v. 15, n. 1, p. 5-24, mar. 2017.

FAZENDA, Ivani Catarina Arantes. Integração e interdisciplinaridade no ensino brasileiro: efetividade ou ideologia. São Paulo: Edições Loyola, 2011.

FREIRE, Paulo. Pedagogia da autonomia: saberes necessários à prática educativa. 57 . ed. Rio de Janeiro: Paz e Terra, 2016.

GAROFALO, Débora. Como levar o STEAM para a sala de aula. Revista Nova Escola, 2019. Disponivel em https://novaescola.org.br/conteudo/18021/como-levar-o-steam-para-a-sala-deaula; acesso em 29 abr. 2020, às 17 h45.

GÓES, Anderson Roges Teixeira; GÓES, Heliza Colaço. Ensino da Matemática: concepções, metodologias, tendências e organização do trabalho pedagógico. Curitiba: InterSaberes, 2015.

GÓES, Anderson Roges Teixeira; GÓES, Heliza Colaço. Modelagem Matemática: teoria, pesquisas e práticas pedagógicas. Curitiba: InterSaberes, 2016.

GÓES, Anderson Roges Teixeira; LUZ, Adriana Augusta Benigno dos Santos. Maquete: uma experiência no ensino da Geometria Plana e Espacial. In: SIMPÓSIO NACIONAL DE GEOMETRIA 
DESCRITIVA E DESENHO TÉCNICO, 19, 2009; INTERNACIONAL CONFERENCE ON GRAPHICS ENGINEERING FOR ARTS AND DESIGN, 8, 2009, Bauru. Anais... Bauru: UNESP, 2009, p. 817-827.

GÓES, Heliza Colaço. Um esboço de conceituação sobre Expressão Gráfica. Revista Educação Gráfica, Bauru, v. 17, n. 1, p. 1-21, jan./abr. 2013.

HOUAISS, Antônio; VILLAR, Mauro de Salles. Dicionário Houaiss da Língua Portuguesa. Elaborado pelo Instituto Antônio Houaiss de Lexicografia e Banco de Dados da Língua Portuguesa S/C Ltda. Rio de Janeiro: Objetiva, 2009.

KENSKI. Vani Moreira. Educação e Tecnologias: o Novo ritmo da informação. Campinas: Papirus, 2012.

LORENZIN, Mariana; ASSUMPÇÃO, Cristiana Mattos; BIZERRA, Alessandra. Desenvolvimento do currículo STEAM no Ensino Médio: a formação de professores em movimento. In: BACICH, Lilian; MORAN, José. (Org.). Metodologias ativas para uma educação inovadora: uma abordagem téorico-prática. Porto Alegre: Penso, 2018, p. 199-219.

RILEY, Susan. STEAM point: a guide to integrating Science, Technology, Engineering, the Arts, and Mathematics through the Common Core. Westminster: EducationCloset, 2012.

VASQUEZ, Jo Anne; SNEIDER, Cary; COMER, Michael. Stem Lesson Essentials, Grades 3-8: integrating Science, Technology, Engineering, and Mathematics. Portsmouth: Heinemann, 2013. 